\title{
Fibroblast Growth Factor-2 Enhances Expansion of Human Bone Marrow-Derived Mesenchymal Stromal Cells without Diminishing Their Immunosuppressive Potential
}

\author{
Jeffery J. Auletta, ${ }^{1}$ Elizabeth A. Zale, ${ }^{2}$ Jean F. Welter, ${ }^{3}$ and Luis A. Solchaga ${ }^{4,5}$ \\ ${ }^{1}$ Divisions of Pediatric Hematology/Oncology and Pediatric Infectious Diseases, Department of Pediatrics, \\ University Hospitals Case Medical Center, Cleveland, OH 44106, USA \\ ${ }^{2}$ Hathaway Brown School, 19600 North Park Boulevard, Shaker Heights, OH 44122, USA \\ ${ }^{3}$ Skeletal Research Center, Department of Biology, Case Western Reserve University, Cleveland, OH 44106-7080, USA \\ ${ }^{4}$ Division of Hematology and Oncology, Department of General Medical Sciences, \\ Case Western Reserve University School of Medicine, Cleveland, OH 44106, USA \\ ${ }^{5}$ BioMimetic Therapeutics, Inc., Franklin, TN 37067, USA
}

Correspondence should be addressed to Luis A. Solchaga, soltxy@gmail.com

Received 18 July 2010; Accepted 13 January 2011

Academic Editor: Amin Rahemtulla

Copyright (C 2011 Jeffery J. Auletta et al. This is an open access article distributed under the Creative Commons Attribution License, which permits unrestricted use, distribution, and reproduction in any medium, provided the original work is properly cited.

\begin{abstract}
Allogeneic hematopoietic stem cell transplantation is the main curative therapy for many hematologic malignancies. Its potential relies on graft-versus-tumor effects which associate with graft-versus-host disease. Mesenchymal stromal cells (MSCs) possess immunomodulatory properties that make them attractive therapeutic alternatives. We evaluated the in vitro immunosuppressive activity of medium conditioned by human MSCs from 5 donors expanded 13 passages with or without FGF-2. FGF-2 supplementation increased expansion 3,500- and 240,000-fold by passages 7 and 13, respectively. There were no differences in immunosuppressive activity between media conditioned by passage-matched cells expanded under different conditions, but media conditioned by FGF-treated MSCs were superior to population doubling-matched controls. The immunosuppressive activity was maintained in three of the preparations but decreased with expansion in two. The proliferation induced by FGF-2 did not result in loss of immunosuppressive activity. However, because the immunosuppressive activity was not consistently preserved, caution must be exercised to ensure that the activity of the cells is sufficient after extensive expansion.
\end{abstract}

\section{Introduction}

Allogeneic hematopoietic stem cell transplantation (HSCT) is the only curative therapy for many hematologic malignancies. Part of the therapeutic benefit of allogeneic HSCT is based on graft-versus-tumor (GVT) effects, which eradicate residual malignant cells through immunologic mechanisms. Unfortunately, GVT is closely associated with the development of graft-versus-host disease (GVHD) [1], which is one of the primary causes of transplant-related mortality [2]. Less than half of patients that develop severe acute GVHD respond to first-line corticosteroid therapy $[3,4]$. Patients with steroid-resistant GVHD require secondary therapy to which only half of these patients respond and have overall poor survival $[2,5]$. Therefore, new therapeutic approaches to prevent and treat GVHD are urgently needed. Cellular therapies are emerging as promising approaches to treat and even to prevent immune-aberrant diseases such as GVHD.

Mesenchymal stromal cells (MSCs) are nonhematopoietic multipotent cells capable of differentiating into both mesenchymal and nonmesenchymal lineages [6-8]. MSCs also produce cytokines, chemokines, and extracellular matrix proteins that support in vitro hematopoietic stem cell (HSC) survival and proliferation and facilitate in vivo HSC engraftment [9]. A substantial body of evidence suggests that MSCs are capable of inhibiting T-lymphocyte activation and proliferation in vitro [10-14]. Additionally, MSCs appear to be immunoprivileged and to escape immune surveillance 
eliciting only weak responses upon rechallenges $[15,16]$. These characteristics make MSCs very attractive as cellular therapeutic agents $[17,18]$.

Human MSC (hMSC) preparations have significant, though variable and finite, proliferative potential [19]. This variability may be due to exogenous factors such as the method used to obtain the bone marrow [20-24], specifics of the serum lots used, and intrinsic factors such as the age of the donor $[21,25]$. Despite their high proliferative potential, extensive culture expansion may result in loss of differentiation potential and the onset of senescence [21]. Interestingly, the senescence-related loss of potential is not generalized; for example, MSCs maintain their osteogenic potential through extensive subcultivation $[20,21]$, but lose their ability to differentiate into adipocytes [21] as they approach senescence. Their ability to differentiate into chondrocytes is lost in earlier passages [26].

Several published reports have demonstrated that fibroblast growth factor-2 (FGF-2) exerts a significant mitogenic effect on MSCs while, simultaneously, enhancing their trilineage (bone, cartilage, fat) differentiation capacity [27-32]. Chondrogenesis, perhaps the differentiation potential most easily lost in MSCs during in vitro culture, is particularly improved by expansion in FGF-2-supplemented medium $[30,31]$.

Little is known, however, about the maintenance or loss of the immunomodulatory activity of hMSCs through extensive expansion or the impact that FGF-2 supplementation might have on this MSC property. The purpose of these experiments was to characterize the immunosuppressive activity of MSCs expanded in vitro for different periods of time with and without FGF-2 supplementation which, as stated above, has been shown to be beneficial for the maintenance of other hMSC functions.

\section{Material and Methods}

All cells were isolated from normal healthy human donors at the Hematopoietic Stem Cell Core Facility of the Comprehensive Cancer Center of Case Western Reserve University after informed consent was obtained under the terms of an Institutional Review Board-approved protocol. Peripheral blood mononuclear cells (PBMCs) were isolated from peripheral blood collected into heparinized blood collection tubes (BD, Franklin Lakes, NJ). Human MCSs were isolated from bone marrow aspirates obtained from the posterior superior iliac crest into a preheparinized $20-\mathrm{mL}$ syringe (BD). The PBMCs and hMSCs used in these studies were isolated from different, unrelated donors.

2.1. Isolation of Human PBMCs. Ten human PBMC preparations were used in these studies. The blood was carefully layered on top of Ficoll (GE Healthcare, Pisctatway, NJ) and the tubes centrifuged at $800 \times \mathrm{g}$ for 30 minutes without brake. After centrifugation, a sterile plastic pipette was used to aspirate the mononuclear cell layer and transfer it into a fresh $50 \mathrm{~mL}$ conical tube (BD). The PBMCs were then washed twice with phosphate buffered saline (PBS, Invitrogen, Carls- bad, CA), counted, and resuspended in complete Roswell Park Memorial Institute (RPMI) 1640 medium composed of RPMI 1640 (Invitrogen) supplemented with 10\% heatinactivated fetal bovine serum (FBS, Invitrogen).

2.2. Isolation of Human MSCs. Five hMSC preparations were used in this study. The procedures for establishing human bone marrow-derived MSC cultures followed previously published methods [33, 34]. Briefly, bone marrow aspirates were washed with control medium consisting of low glucose Dulbecco's modified Eagle's medium (DMEM-LG, Invitrogen) supplemented with $10 \%$ fetal bovine serum (FBS) from a selected lot (Hyclone, Logan, UT) [34]. Serum lot selection is a standard procedure performed prior to purchasing a new shipment of serum; all experiments were conducted with serum from a single lot. They were then centrifuged on a Percoll (Sigma Chemical Co., St. Louis, MO) density gradient to isolate mononuclear cells. The mononuclear cells were washed with control medium and seeded at a density of $1.8 \times 10^{5} \mathrm{cells} / \mathrm{cm}^{2}$ in control medium to establish primary cultures. All cell culture was done at $37^{\circ} \mathrm{C}$ in a humidified atmosphere of $95 \%$ air and $5 \% \mathrm{CO}_{2}$.

2.3. Establishment of Study Groups. At the first medium change (day 4), and in every medium change thereafter, some of the plates received control medium, and the rest of the plates received the same medium supplemented with $10 \mathrm{ng} / \mathrm{mL}$ of FGF-2 (Peprotech, Rocky Hill, NJ). The dose was chosen based on previous studies [31]. Cultures were fed twice per week.

2.4. Expansion of hMSCs. hMSCs must be subcultured before the cells become confluent in order to keep their growth at an exponential rate and prevent spontaneous differentiation or loss of differentiation potential $[6,35]$. Typically, they were passaged when the cultures were $80-90 \%$ confluent. Primary cultures were usually subcultured at 14 \pm 3 days. Subsequently, the cells were subcultured approximately every $7 \pm 2$ days. Plates assigned to the different study groups (control or FGF-treated) were subcultured at the same time, which resulted in different levels of confluence in the treatment groups as result of the previously reported differences in cell proliferation and cell size [31]. In all cases, control cultures were less confluent than their FGFtreated counterparts at the time of subcultivation. Cells were subcultured by trypsinization, counted, and reseeded at 4.5 $\times 10^{3}$ cells per $\mathrm{cm}^{2}$.

2.5. Cryopreservation of hMSCs. Subcultured cells were centrifuged at $200 \times \mathrm{g}$ for 5 minutes; the supernatant was discarded, and the cells resuspended in cold freezing medium consisting of $90 \%$ FBS (Invitrogen) and 10\% DMSO (Sigma Chemical Co.) at a density of $10^{6}$ cells $/ \mathrm{mL}$. The cells were aliquoted into cryogenic storage vials (Thermo Fisher Scientific, Rochester, NY), the vials placed in a freezing container (Thermo Fisher Scientific), and the container placed at $-80^{\circ} \mathrm{C}$ overnight. The vials were then transferred to the vapor phase of a liquid nitrogen freezer. 
2.6. Recovery of Cryopreserved hMSCs. Vials containing approximately $1.0-1.5 \times 10^{6}$ cryopreserved cells were thawed quickly in a $37^{\circ} \mathrm{C}$ water bath and transferred into a 15 $\mathrm{mL}$ conical tube containing $5 \mathrm{~mL}$ of either control or FGFsupplemented medium, as appropriate. The tubes were then centrifuged at $200 \times \mathrm{g}$ for 5 minutes. After centrifugation the supernatant was discarded, and the cells were resuspended in either control or FGF-supplemented medium and seeded at $4.5 \times 10^{3}$ cells per $\mathrm{cm}^{2}$.

2.7. Characterization of hMSCs. The hMSC phenotype was confirmed by forward and side-scatter pattern as well as monoclonal antibody (MAb) staining $\left(\mathrm{CD} 45^{-}\right.$, $\left.\mathrm{CD}^{+} 3^{+}, \mathrm{CD} 105^{+}\right)$. Briefly, approximately 500,000 hMSCs were washed and resuspended in HBSS supplemented with $1 \%$ FBS containing $5 \mathrm{mM}$ EDTA. hMSCs were then incubated with fluorescein isothiocyanate (FITC)-conjugated anti-CD45 MAb (BD), phycoerythrin (PE)-conjugated antiCD73 MAb (BD), and allophycocyanin (APC)-conjugated anti-CD105 MAb (eBioscience, San Diego, CA). After 30minute incubation at $4^{\circ} \mathrm{C}$, MAb-labeled cells were washed three times and then fixed in 2\% paraformaldehyde prior to analysis. Isotype control immunoglobulins were used as negative controls for staining. At least 20,000 events were analyzed per conjugated MAb stain condition.

To assess their chondrogenic potential [36-38], passaged cells were resuspended in a chemically defined chondrogenic medium. Aliquots containing $2.5 \times 10^{5}$ cells were placed in polypropylene multiwell plates, centrifuged at $500 \times \mathrm{g}$, and placed in the incubator. Medium was changed three times per week. On days 7, 14, and 21 aggregates were harvested and processed for histologic evaluation.

To verify their osteogenic potential $[20,39,40]$ hMSCs were seeded at a density of $5 \times 10^{3}$ cells $/ \mathrm{cm}^{2}$ in control medium and allowed to attach overnight. The next day, the culture medium was replaced with osteogenic medium. Culture medium is changed twice per week. Osteogenic cultures are further supplemented with $2 \mathrm{mM} \beta$-glycerophosphate starting on day 10. Triplicate cultures were stained for calcium deposition (von Kossa) [41] on days 14, 21, and 28.

The adipogenic potential of hMSCs was also tested [42]; passaged cells aliquots containing $2.5 \times 10^{5}$ cells were placed in polypropylene multiwell plates in adipogenic induction medium in multiwell plates, centrifuged at $500 \times \mathrm{g}$, and placed in the incubator. Medium was changed three times per week. On day 10, the medium was replaced with adipogenic maintenance medium. Cells were harvested for histologic analysis on days 7,14 , and 21 .

2.8. Generation of hMSC-Conditioned Media. hMSCs at different passage numbers were seeded into 6-well plates (BD), at a density $15 \times 10^{3} \mathrm{cells} / \mathrm{cm}^{2}$ in complete hMSC medium. After overnight incubation, the medium was removed and a 2-mL aliquot of complete RPMI containing either PBMCs $\left(0.5 \times 10^{6}\right.$ cells $\left./ \mathrm{mL}\right)$ or interleukin-1 beta (IL$1 \beta)(5 \mathrm{pg} / \mathrm{mL}$, Peprotech) was added to each well. Control wells received only complete RPMI. Another set of control wells was incubated without hMSCs, but still received either
PBMCs or IL-1 $\beta$. After a 24 -hour incubation period, the conditioned media were collected into 2 -mL microcentrifuge tubes and centrifuged for 10 minutes at $12,000 \times \mathrm{g}$ to remove any remaining cells. The cell-free supernatants were then transferred to clean microcentrifuge tubes and either used fresh or frozen at $-80^{\circ} \mathrm{C}$ for later use.

2.9. Assessment of Cell Expansion. All cell counts during the expansion phase were done manually on the trypsinized cell suspensions, in triplicate using a Neubauer hemocytometer. Population doublings were calculated as the base- 2 logarithm of the number of cells obtained at the end of a given passage divided by the number of cell seeded.

For primary cultures, the number of colonies formed in primary culture we used as the denominator assuming that one colony is derived from one MSC.

2.10. Interferon-Gamma ELISpot Assay. The immunosuppressive activity of hMSCs and their conditioned media was tested in enzyme-linked immunosorbent spot assays (ELISpot) [43]. The ELISpot assay allows visualization of the secretory product of individual responding cells; each spot that develops in the assay represents a single reactive cell. Thus, the assay provides both qualitative (type of immune protein) and quantitative (number of responding cells) information. ELISpot assays are highly sensitive because the product is rapidly captured around the secreting cell before it is diluted in the supernatant, captured by receptors of adjacent cells, or degraded. The assay has gained a recent increase in popularity, especially as a surrogate measure for cytotoxic T-cell responses in large part because it is both reliable and highly sensitive [44].

Ninety-six well ELISpot plates (Millipore, Billerica, MA) were coated with antihuman interferon-gamma (IFN $\gamma$ ) antibody (Pierce, Rockford, IL); $100 \mu \mathrm{L}$ of antibody solution $(4 \mu \mathrm{g} / \mathrm{mL}$ in PBS) were added to each of the 96 wells of the plate and incubated overnight in the refrigerator. The plate was then washed with PBS and blocked at $37^{\circ} \mathrm{C}$ for 2 hours with complete RPMI. The wells then received either $150 \mu \mathrm{L}$ of complete RPMI (control wells) or $150 \mu \mathrm{L}$ of either a $10^{6}$ cells/mL hMSC suspension or hMSCconditioned medium (experimental wells). Then, $25 \mu \mathrm{L}$ of complete RPMI were added to the negative control wells, and $25 \mu \mathrm{L}$ of phytohemagglutinin (PHA, Sigma Chemical Co) solution $(40 \mu \mathrm{g} / \mathrm{mL}$ in complete RPMI) were added to experimental and positive control wells. A $25-\mu \mathrm{L}$ aliquot of PBMC suspension $\left(6 \times 10^{6}\right.$ cells $\left./ \mathrm{mL}\right)$ was finally added to each well, and the plate was incubated for 24 hours at $37^{\circ} \mathrm{C}$. After the incubation, the plate was washed with PBS $+0.05 \%$ Tween 20 (Sigma Chemical Co.). Biotinylated antiIFN $\gamma$ antibody (Pierce) $(2 \mu \mathrm{g} / \mathrm{mL}$ in PBS $+0.05 \%$ Tween $20+1 \%$ BSA (Sigma Chemical Co.) was added and the plate incubated at $37^{\circ} \mathrm{C}$ for 2 hours. After washing the plate with PBS $+0.05 \%$ Tween 20, Streptavidin-Horseradish Peroxidase (Dako, Glostrup, Denmark) diluted 1:1,000 in PBS $+0.05 \%$ Tween $20+1 \%$ BSA was added and the plate incubated for 1 hour. After 3 washes with PBS $+0.05 \%$ Tween 20 followed by 4 washes with PBS the IFN $\gamma$-positive 


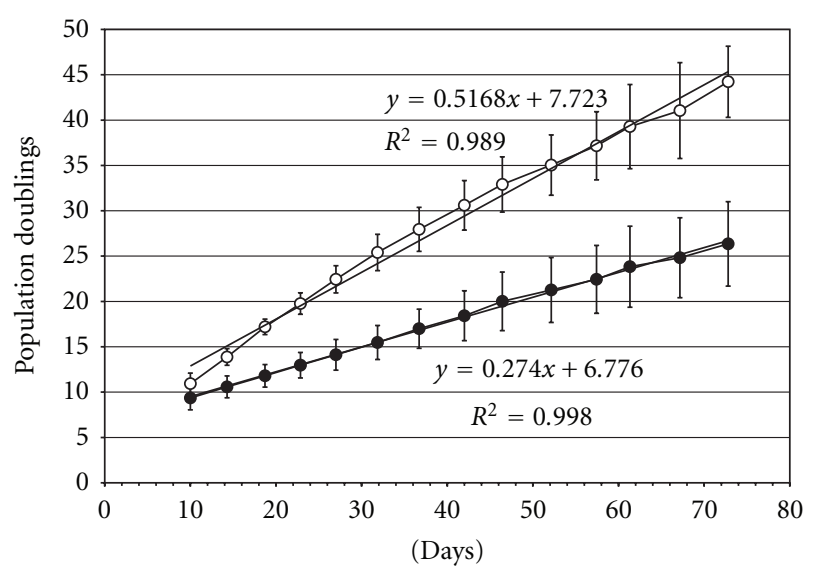

Figure 1: Culture Expansion. Cumulative population doublings of hMSCs expanded in control conditions (black circles) or in the presence of rhFGF-2 (white circles). Mean \pm standard deviation from 5 individual hMSC preparations. The slopes of the linear regression equations are an approximation to the average population doubling times.

spots were developed with 3-amino-9-ethyl carbazole (AEC) (Pierce). The reaction was then stopped with tap water, and the plates were allowed to dry in the dark. The plates were analyzed with a computer-assisted ELISpot analyzer (Cellular Technology Inc., Cleveland, $\mathrm{OH}$ ). Percent inhibition was obtained by direct comparison to the corresponding positive control indicated above.

2.11. Statistical Analyses. The statistical significance of the differences in proliferation rates and of the differences between PBMC and IL- $1 \beta$ stimulation was determined by paired $t$-tests. Analysis of the effect of time-in-culture on the immunosuppressive activity was performed by one-factor repeated measurements ANOVA followed by paired $t$-tests to identify the differences between passages. The analysis of the effect of FGF supplementation was performed by paired $t$-tests, and differences were considered significant for $P$ values $<.05$.

\section{Results}

3.1. Cell Expansion. As in previous studies, hMSCs expanded in the presence of FGF-2 exhibited higher proliferation rates than those expanded in control conditions [31]. The population doubling time for hMSCs expanded in the presence of FGF-2 was consistently shorter than that of cells expanded under control conditions at any passage $(P<.01)$ as indicated by the slope of the growth curves in Figure 1. By passage 13, control MSCs reached on average $26.3 \pm$ 4.7 population doublings (PDs), while FGF-treated cells reached $44.2 \pm 3.9$ PDs. FGF-treated hMSCs reached 28.0 \pm 2.6 PDs (the maximum expansion obtained with control cells) around passage 6 in approximately 35 days. Control or FGF-treated cells were subcultured at the same time, causing different levels of confluence, a consequence of the differences in cell proliferation and cell size [31]. Control cultures were never more confluent than their FGF-treated counterparts at any time of subcultivation.

3.2. Cell Characterization. hMSCs isolated from bone marrow exhibited the typical characteristics of multipotent hMSCs [45], that is, characteristic morphology, surface marker profile (not shown), and trilineage differentiation potential (Figure 2).

3.3. Assessment of the Immunomodulatory Activity of hMSCConditioned Medium. Conditioned medium from PBMCstimulated hMSCs exhibited higher activity than the PBMCstimulated hMSCs themselves (one-tail $t$-test; $P=5.52 \times$ $\left.10^{-7} ; n=5\right)$, as measured by decreases in the number of IFN $\gamma$-positive spots. The immunosuppressive potential of PBMC-stimulated hMSCs and their conditioned media was higher (one-tail $t$-test; $P=1.46 \times 10^{-7} ; n=5$ ) than that of the conditioned media from nonstimulated hMSCs and the non-stimulated hMSCs themselves (Figure 3). Supported by these results and for logistical reasons and ease of use, indepth analysis of the immunosuppressive activity of hMSCs from the study groups at different passages was performed on conditioned media samples rather than with the cells.

3.4. Stimulation of hMSCs by $I L-1 \beta$. Conditioned media generated after activation of hMSCs with either PBMCs or IL- $1 \beta$ exhibited immunosuppressive activity in IFN $\gamma$ ELISpots (Figure 4). The conditioned media generated by stimulation of hMSCs with IL- $1 \beta$ exhibited higher (one-tail paired $t$-test; $\left.P=5.23 \times 10^{-7}, n=50\right)$ and more consistent immunosuppressive activity $(\mathrm{CV}=33 \%)$ than media from cultures stimulated with PBMCs $(\mathrm{CV}=54 \%)$.

The higher variability of the conditioned media generated by stimulation of hMSC cultures with PBMCs likely reflects the interindividual variability among the blood donors from whom the PBMCs were isolated.

Consequently, for simplicity and data consistency, we focused our analyses on conditioned media from IL- $1 \beta$ stimulated hMSCs although both PBMC and IL- $1 \beta$ stimulation were tested for all cultures.

3.5. Effect of Ex Vivo Expansion on the Immunosuppressive Potential of hMSCs. To analyze the effect of time-in-culture on the immunosuppressive potential of each hMSC preparation, the inhibitory activity of each passage was normalized to that of the first passage of that preparation to minimize variability in the intrinsic degree of inhibition among the 5 cell preparations.

Overall, when all five cell preparations were considered, there was a decrease in the immunosuppressive activity as a function of time in culture (single factor ANOVA; $P=$ $.010, n=10$ ) (Figure 5(a)). Conditioned media from cells at passage 1 and passage 4 had equivalent activity (one-tail paired $t$-test; $P=.437, n=10$ ) to one another and superior to the activity of conditioned media from cells at passages 7,10 , and 13 (one-tail paired $t$-test; $P \leq .044, \quad n=10$ ); conditioned media from cells at passage 7 had similar activity 


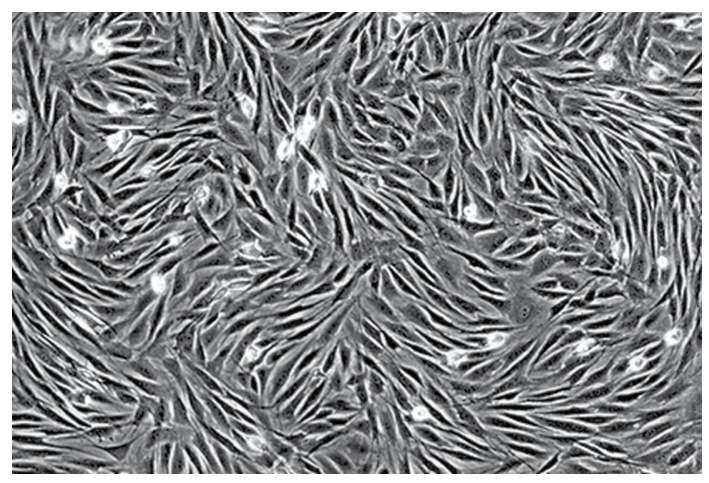

(a)

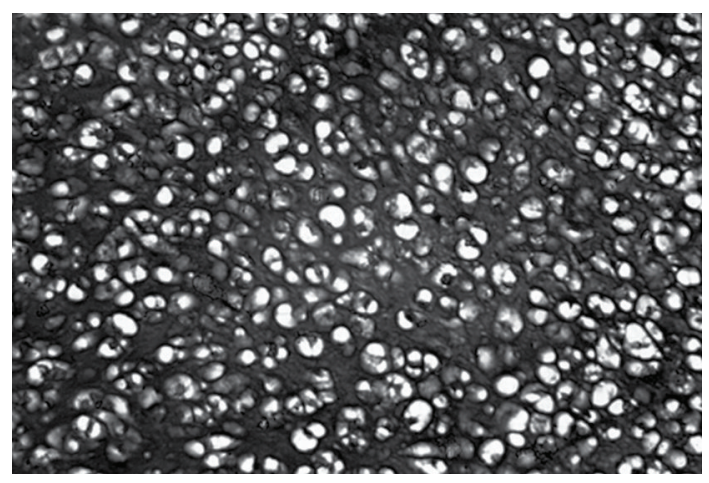

(c)

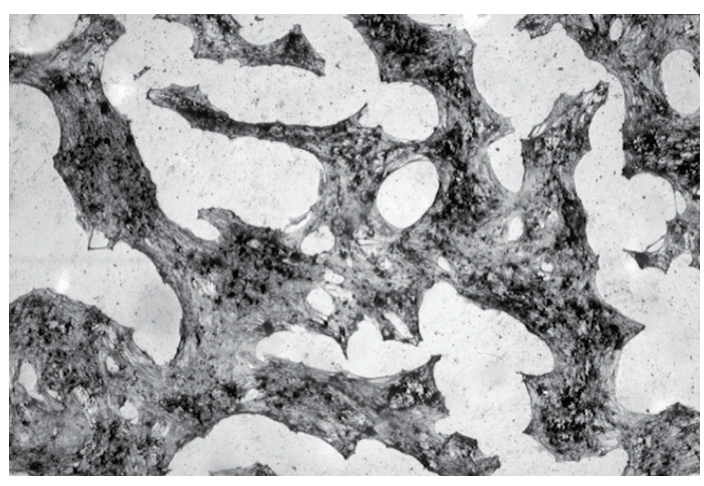

(b)

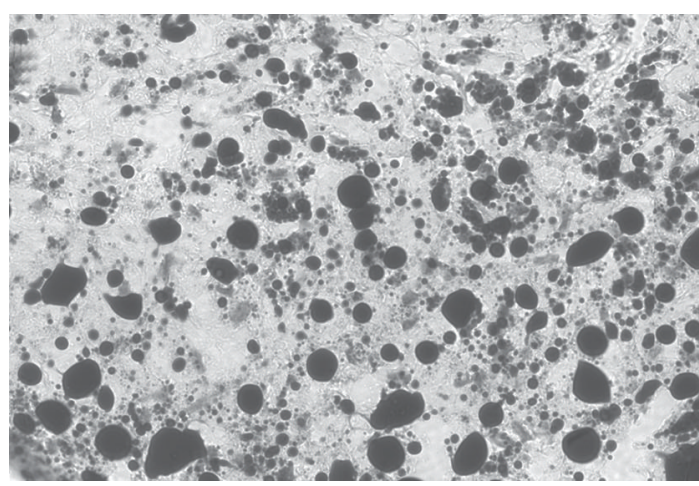

(d)

Figure 2: Functional characterization of hMSCs. Human MSCs (a) in culture, (b) after 4 weeks in osteogenic conditions (von Kossa staining), (c) after 3 weeks in chondrogenic conditions (toluidine blue staining), and (d) after 3 weeks in adipogenic conditions (oil-red O staining).

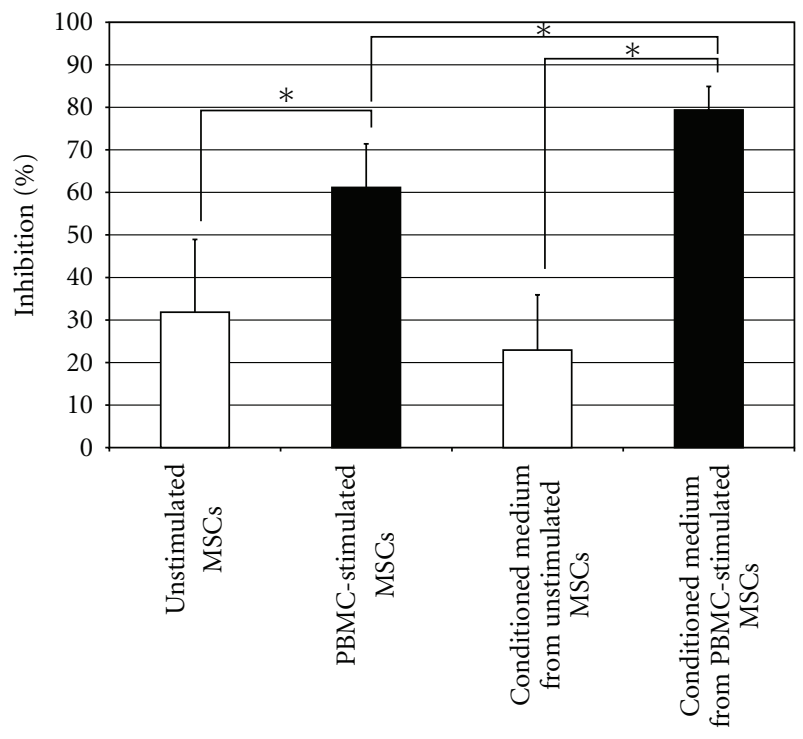

FIGURE 3: Immunosuppressive activity of hMSC-conditioned medium. Immunosuppressive activity of unstimulated (white bars) and PBMC-stimulated (black bars) hMSCs and hMSC-conditioned media. Values are expressed as percent inhibition relative to PHAstimulated PBMCs which served as controls. Higher bars indicate more immunosuppressive activity. Asterisks indicate significant differences in one-tail $t$-test; ${ }^{*} P<.01$. to that of cells from passages 10 and 13 (one-tail paired $t$ test; $P \geq .146, \quad n=10$ ), and conditioned media from cells at passage 10 were more immunosuppressive than those of cells from passage 13 (one-tail paired $t$-test; $P=.007, \quad n=10$ ).

In three of the cell preparations tested, the immunosuppressive activity was unchanged over the passages tested (single factor ANOVA; $P=.064, n=6$ ) (Figure 5(b)) while in two preparations the activity exhibited a continued decrease over time (single factor ANOVA; $P=.017, n=4$ ) (Figure 5(c)).

3.6. Effect of FGF-2 Supplementation on the Immunosuppressive Potential of hMSCs. No significant differences were observed between the immunosuppressive activity of cell preparation- and passage-matched hMSCs grown under control conditions or supplemented with FGF-2 (one-tail paired $t$-test; $P=.285 ; n=25$ ) (Figure 6(a)). However, the comparison of passage-matched groups may not be biologically relevant. As indicated above, the number of population doublings that FGF-treated cells have undergone at any given passage is higher than those of their control counterparts. When the comparison between the two different culture conditions was performed matching the subpopulations within each cell preparation by the number of population doublings rather than by the number of passages, cells 


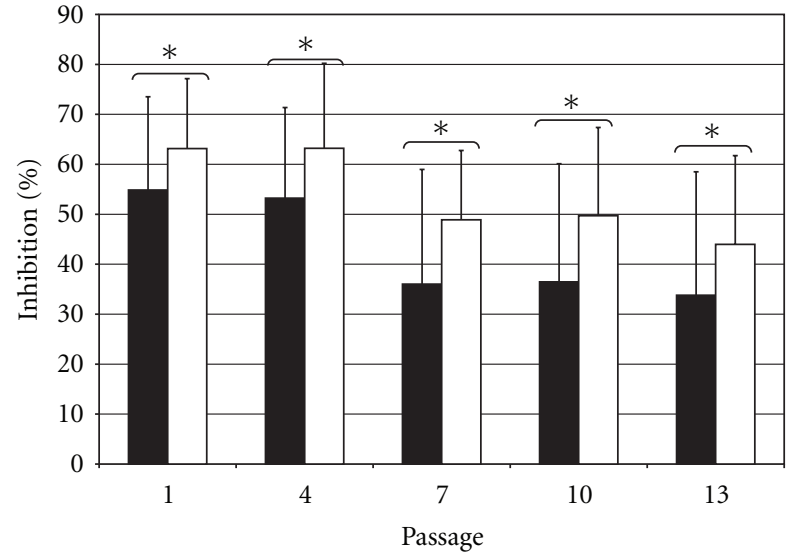

Figure 4: Effect of PBMC and IL- $1 \beta$ stimulation on the immunosuppressive activity of hMSCs. Immunosuppressive activity (mean \pm standard deviation; $n=10 ; 5$ control and 5 FGF-treated) of medium conditioned by MSCs from different passages stimulated with PBMCs (black bars) or IL-1 $\beta$ (white bars). Values are expressed as percent inhibition relative to the internal controls. Higher bars indicate higher immunosuppressive activity. Asterisks indicate significant differences in one-tail paired $t$-test; ${ }^{*} P<.05$.

expanded in FGF-supplemented medium exhibited higher immunosuppressive activity than their control counterparts (one-tail paired $t$-test; $P=.002 ; n=11$ ) (Figure 6(b)).

\section{Discussion}

Due to their immunosuppressive properties, MSCs are being tested clinically to treat GVHD and other autoimmune disorders [18, 46-49]. However, because of the low frequency of MSCs in the bone marrow [50], the clinical application of hMSCs to treat GVHD requires extensive ex vivo expansion to achieve the cell doses currently utilized for patient treatment. For example, for an $80-\mathrm{kg}$ patient, one single hMSC injection at the lowest dose $\left(10^{6}\right.$ cells per $\mathrm{kg}$ of body weight) being tested in clinical trials would necessitate 8 $\times 10^{7}$ cells. In this dataset, that level of expansion would have been reached by the end of the fourth passage under control conditions or by the end of the first passage under FGF-supplemented conditions. If multiple injections and/or higher doses were required, the cell number needed for the entire course of treatment could be as high as $4 \times 10^{9}$ cells. In the current experiments, this higher level of expansion would require 7 or 8 passages in control and 4 passages in FGF-supplemented conditions.

The data presented here confirm the value of expansion in FGF-supplemented medium [31, 51-53]. Specifically, compared to expansion in control conditions, FGF-2 supplementation would result, on average, in 3,500-fold increase in the number of hMSCs obtained by passage 7, 24,000fold increase in hMSCs by passage 10, and 240,000-fold increase in hMSCs by passage 13. These differences can be critical in the clinical application of these cells, as FGF supplementation can expedite production of cells resulting in more rapid preclinical testing, characterization, and

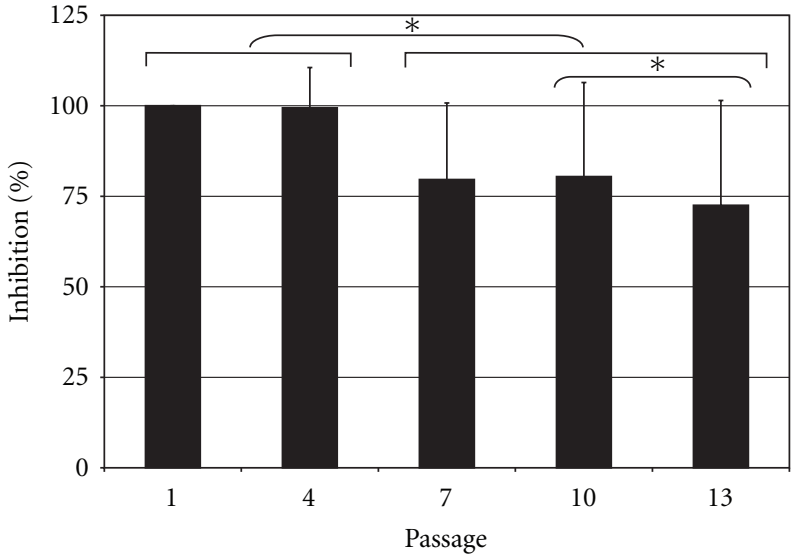

(a)

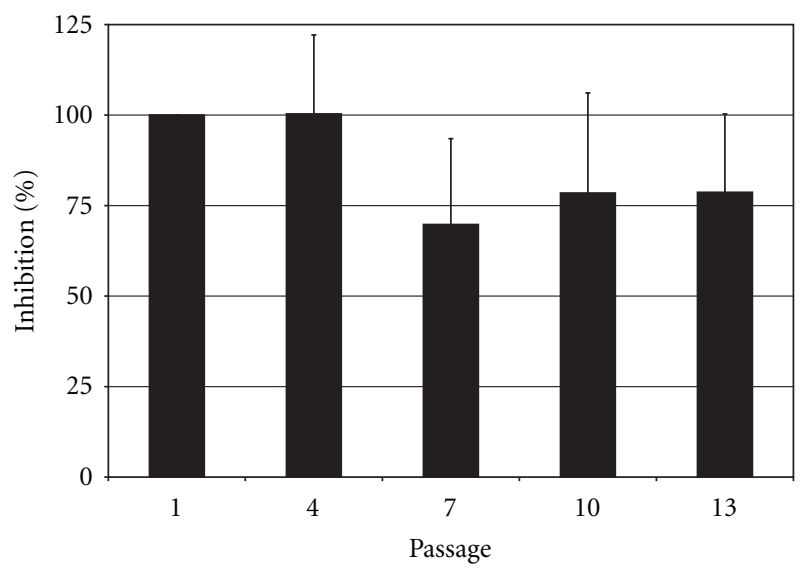

(b)

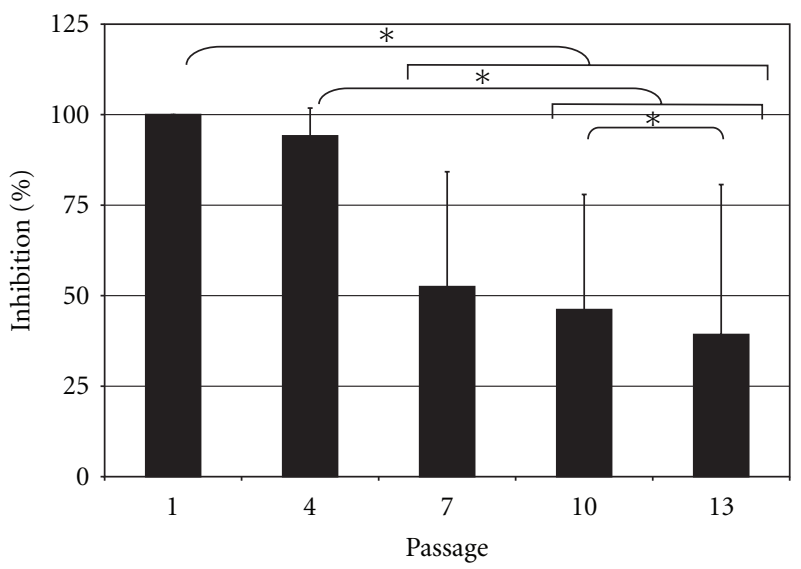

(c)

FIGURE 5: Effect of time-in-culture on the immunosuppressive activity of hMSCs. Immunosuppressive activity in conditioned medium from IL- $1 \beta$-stimulated hMSCs at different passages cultured under control conditions. Values are normalized to the inhibition observed conditioned medium from first passage hMSCs. The mean and standard deviation for all 5 hMSC preparations are displayed in (a); the 3 hMSC preparations that exhibited sustained activity are displayed in (b); the mean and standard deviation for the 2 hMSC preparations that exhibited decreased activity after 4 passages are displayed in (c). Asterisks indicate significant differences in one-tail paired $t$-test; ${ }^{*} P<.05$. 


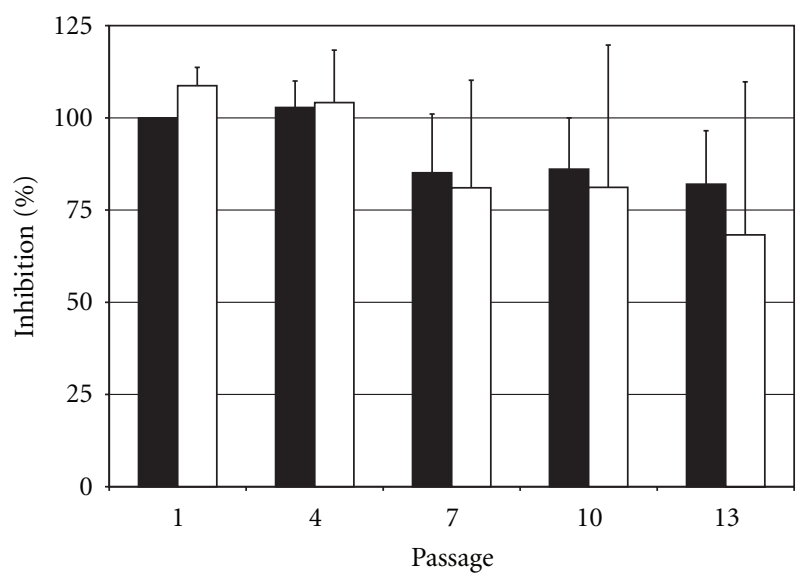

(a)

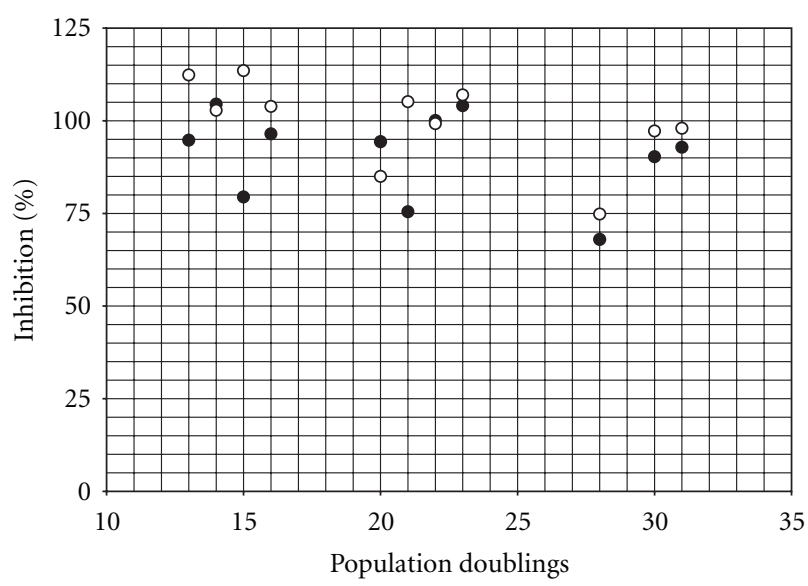

(b)

FIGURE 6: Effect of culture conditions on the immunosuppressive activity of hMSCs. Immunosuppressive activity of medium conditioned by IL- $1 \beta$-stimulated hMSCs expanded in control or FGFsupplemented medium. Values are normalized to the inhibitory activity of first passage hMSCs expanded in control conditions. (a) Immunosuppressive activity (mean \pm standard deviation; $n=5$ ) of medium conditioned by IL- $1 \beta$-stimulated hMSCs from different passages expanded in control (black bars) or FGF-supplemented (white bars) medium. (b) Immunosuppressive activity of medium conditioned by IL- $1 \beta$-stimulated hMSCs expanded in control (black circles) or FGF-supplemented (white circles) medium. The data are presented as pairs of preparation- and passage-matched hMSCs subsets. Note that for 8 of the 11 data pairs $(73 \%)$ the conditioned medium from FGF-treated hMSCs is more immunosuppressive than that of their control counterparts. Overall, the differences in immunosuppressive activity between conditioned media from control and FGF-treated cells are significant (one-tail paired $t$-test; $P=.002$ ).

availability of clinical-grade hMSCs, thereby significantly impacting the clinical application of MSC therapies.

It is well known that hMSC preparations have significant proliferative potential [19] but their extensive expansion results in loss of differentiation potential, onset of senescence [21], and/or apoptosis [54]. Although reports in the literature provide ample support for the immunosuppressive activity of hMSCs $[10,13,14,17,55-57]$, the fate of this activity as the cell preparations are expanded has not been studied in depth. To date, only one report indicates that the immunosuppressive activity of hMSCs does not appear to decrease through 6 or 7 passages, as measured in vitro [58].

Our studies included the standard expansion conditions as initially described by Haynesworth and colleagues in 1992 [59] and supplementation with FGF-2 which has been shown to have beneficial effects on the proliferative and differentiation potentials of MSCs [27-29, 31, 32, 60]. We expanded the cell preparations for 13 passages, a level of expansion at which most hMSC preparations have reached senescence [19]. In this expansion, control MSCs reached $26.3 \pm 4.7$ population doublings by passage 13 , while FGFtreated cells reached $44.2 \pm 3.9$. Of note, FGF-treated hMSCs reached $28.0 \pm 2.6$ population doublings (the maximum expansion obtained with control cells) around passage 6 in approximately 5 weeks compared to the 9 weeks required to achieve that level of expansion in control conditions, a difference that might be crucial for the timely administration of the cellular product.

FGF-treated hMSCs exhibited immunosuppressive activity comparable to that of passage-matched controls and that of population doubling-matched controls. Thus, while FGF supplementation does not elicit the significant improvements that have been reported for the chondrogenic potential of hMSCs [31], maintaining the immunosuppressive activity is critical for the application to disease conditions associated with aberrant T-cell alloreactivity.

On a the technical aspect and relative to assay optimization, our data corroborate previous reports indicating that, at least in part, the immunosuppressive activity of hMSCs is mediated through soluble factors $[13,55,61-$ 63 ] and that hMSCs likely need to be stimulated by their microenvironment to exert this activity $[14,63,64]$. We have shown that medium conditioned by MSCs activated with PBMCs is immunosuppressive. This observation allows the use of conditioned medium to characterize the activity of the hMSCs. To this end, conditioned media from different preparations and passages can be prepared and stored and then tested simultaneously against the same effector cells, allowing better comparison of the results.

Additionally, the immunosuppressive activity of conditioned media from hMSC activated with PBMCs or IL- $1 \beta$ was comparable, but media generated with IL- $1 \beta$ had higher activity and, importantly, less variability in immunosuppressive activity, supporting the use of IL- $1 \beta$ instead of PBMCs for activation of the hMSCs and simplifying the preparation of the conditioned media in order to standardize stimulation using a recombinant cytokine rather than uncharacterized cell preparations.

\section{Conclusions}

In summary, compared to control conditions, hMSC expansion in FGF-supplemented medium would potentially benefit the intended recipients of these cellular products by enabling the acquisition of equivalent numbers of cells in a 
significantly reduced timeframe or more cells in the same period of time without loss of their immunosuppressive activity.

However, the immunosuppressive activity as measured in vitro using IFN $\gamma$ ELISpot assays was not universally preserved in all the cell preparations from individual donors. Therefore, caution must be exercised when significant expansion of the MSCs is desired or required. In these cases, the activity of the final cell population should be verified, and perhaps activity or potency minimums should be established as part of the release criteria for therapeutic application. In addition, these initial in vitro observations must be validated using in vivo models because the microenvironment could certainly affect the activity or performance of these cells [65].

\section{Acknowledgments}

The authors would like to thank Dr. Wouter van 't Hof for constructive discussions of the work and Dr. Sylvia Janetzki for technical advice on the ELISpot assays. This research was supported by Grants from the Ohio Department of Development (Center for Stem Cell and Regenerative Medicine Pilot Program; PIs; Luis A. Solchaga and Jeffery J. Auletta), and by the NIH (NIAID K08 A57801; PI: Jeffery J. Auletta and NIAMS R01 AR05028; PI: Jean F. Welter). This work was also supported by the Hematopoietic Stem Cell Core Facility of the Case Comprehensive Cancer Center (NCI; P30 CA43703; PI: Stanton L. Gerson).

\section{References}

[1] D. H. Fowler, "Shared biology of GVHD and GVT effects: potential methods of separation," Critical Reviews in Oncology/Hematology, vol. 57, no. 3, pp. 225-244, 2006.

[2] M. C. Pasquini, "Impact of graft-versus-host disease on survival," Best Practice and Research: Clinical Haematology, vol. 21, no. 2, pp. 193-204, 2008.

[3] I. Yakoub-Agha, F. Mesnil, M. Kuentz et al., "Allogeneic marrow stem-cell transplantation from human leukocyte antigen-identical siblings versus human leukocyte antigenallelic-matched unrelated donors (10/10) in patients with standard-risk hematologic malignancy: a prospective study from the French society of bone marrow transplantation and cell therapy," Journal of Clinical Oncology, vol. 24, no. 36, pp. 5695-5702, 2006.

[4] D. J. Weisdorf, C. Anasetti, J. H. Antin et al., "Allogeneic bone marrow transplantation for chronic myelogenous leukemia: comparative analysis of unrelated versus matched sibling donor transplantation,” Blood, vol. 99, no. 6, pp. 1971-1977, 2002.

[5] J. C. Gea-Banacloche and G. A. Weinberg, "Monoclonal antibody therapeutics and risk for infection," Pediatric Infectious Disease Journal, vol. 26, no. 11, pp. 1049-1052, 2007.

[6] S. E. Haynesworth, J. Goshima, V. M. Goldberg, and A. I. Caplan, "Characterization of cells with osteogenic potential from human marrow," Bone, vol. 13, no. 1, pp. 81-88, 1992.

[7] I. B. Black and D. Woodbury, "Adult rat and human bone marrow stromal stem cells differentiate into neurons," Blood Cells, Molecules, and Diseases, vol. 27, no. 3, pp. 632-636, 2001.
[8] D. Woodbury, E. J. Schwarz, D. J. Prockop, and I. B. Black, "Adult rat and human bone marrow stromal cells differentiate into neurons," Journal of Neuroscience Research, vol. 61, no. 4, pp. 364-370, 2000.

[9] L. M. Ball, M. E. Bernardo, H. Roelofs et al., "Cotransplantation of ex vivo-expanded mesenchymal stem cells accelerates lymphocyte recovery and may reduce the risk of graft failure in haploidentical hematopoietic stem-cell transplantation," Blood, vol. 110, no. 7, pp. 2764-2767, 2007.

[10] K. McIntosh, E. Klyushnenkova, V. Shustova, A. Moseley, and R. Deans, "Suppression of alloreactive T cell response by human mesenchymal stem cells involves CD+ cells," Blood, vol. 94, p. 133a, 1999.

[11] M. D. Nicola, C. Carlo-Stella, M. Magni et al., "Human bone marrow stromal cells suppress T-lymphocyte proliferation induced by cellular or nonspecific mitogenic stimuli," Blood, vol. 99, no. 10, pp. 3838-3843, 2002.

[12] K. Le Blanc, L. Tammik, B. Sundberg, S. E. Haynesworth, and O. Ringdén, "Mesenchymal stem cells inhibit and stimulate mixed lymphocyte cultures and mitogenic responses independently of the major histocompatibility complex," Scandinavian Journal of Immunology, vol. 57, no. 1, pp. 11-20, 2003.

[13] W. T. Tse, J. D. Pendleton, W. M. Beyer, M. C. Egalka, and E. C. Guinan, "Suppression of allogeneic T-cell proliferation by human marrow stromal cells: implications in transplantation," Transplantation, vol. 75, no. 3, pp. 389-397, 2003.

[14] B. Maitra, E. Szekely, K. Gjini et al., "Human mesenchymal stem cells support unrelated donor hematopoietic stem cells and suppress T-cell activation," Bone Marrow Transplantation, vol. 33, no. 6, pp. 597-604, 2004.

[15] M. Sundin, O. Ringdén, B. Sundberg, S. Nava, C. Götherström, and K. Le Blanc, "No alloantibodies against mesenchymal stromal cells, but presence of anti-fetal calf serum antibodies, after transplantation in allogeneic hematopoietic stem cell recipients," Haematologica, vol. 92, no. 9, pp. 1208-1215, 2007.

[16] M. Sundin, A. J. Barrett, O. Ringdén et al., "HSCT Recipients have specific tolerance to MSC but not to the MSC donor," Journal of Immunotherapy, vol. 32, no. 7, pp. 755-764, 2009.

[17] O. Ringdén, M. Uzunel, I. Rasmusson et al., "Mesenchymal stem cells for treatment of therapy-resistant graft-versus-host disease," Transplantation, vol. 81, no. 10, pp. 1390-1397, 2006.

[18] K. Le Blanc, F. Frassoni, L. Ball et al., "Mesenchymal stem cells for treatment of steroid-resistant, severe, acute graft-versushost disease: a phase II study," The Lancet, vol. 371, no. 9624, pp. 1579-1586, 2008.

[19] J. J. Minguell, A. Erices, and P. Conget, "Mesenchymal stem cells," Experimental Biology and Medicine, vol. 226, no. 6, pp. 507-520, 2001.

[20] S. P. Bruder, N. Jaiswal, and S. E. Haynesworth, "Growth kinetics, self-renewal, and the osteogenic potential of purified human mesenchymal stem cells during extensive subcultivation and following cryopreservation," Journal of Cellular Biochemistry, vol. 64, no. 2, pp. 278-294, 1997.

[21] C. M. Digirolamo, D. Stokes, D. Colter, D. G. Phinney, R. Class, and D. J. Prockop, "Propagation and senescence of human marrow stromal cells in culture: a simple colony-forming assay identifies samples with the greatest potential to propagate and differentiate," British Journal of Haematology, vol. 107, no. 2, pp. 275-281, 1999.

[22] D. G. Phinney, G. Kopen, W. Righter, S. Webster, N. Tremain, and D. J. Prockop, "Donor variation in the growth properties and osteogenic potential of human marrow stromal cells," Journal of Cellular Biochemistry, vol. 75, no. 3, pp. 424-436, 1999. 
[23] I. Blazsek, B. D. Marsalet, S. Legras, S. Marion, D. Machover, and J. L. Misset, "Large scale recovery and characterization of stromal cell-associated primitive haemopoietic progenitor cells from filter-retained human bone marrow," Bone Marrow Transplantation, vol. 23, no. 7, pp. 647-657, 1999.

[24] M. Sundin, M. Remberger, H. Lönnies, B. Sundberg, O. Ringdén, and K. Le Blanc, "No increased trapping of multipotent mesenchymal stromal cells in bone marrow filters compared with other bone marrow cells," Cytotherapy, vol. 10, no. 3, pp. 238-242, 2008.

[25] M. Galotto, G. Berisso, L. Delfino et al., "Stromal damage as consequence of high-dose chemo/radiotherapy in bone marrow transplant recipients," Experimental Hematology, vol. 27, no. 9, pp. 1460-1466, 1999.

[26] M. F. Pittenger, G. Mbalaviele, M. Black, J. D. Mosca, and D. R. Marshak, "Mesenchymal stem cells," in Primary Mesenchymal Cells, M. R. Koller, B. O. Palsson, and J. R. W. Masters, Eds., Human cell culture ; v. 5, pp. 189-207, Kluwer Academic Publishers, Boston, Mass, USA, 2001.

[27] A. Banfi, A. Muraglia, B. Dozin, M. Mastrogiacomo, R. Cancedda, and R. Quarto, "Proliferation kinetics and differentiation potential of ex vivo expanded human bone marrow stromal cells: implications for their use in cell therapy," Experimental Hematology, vol. 28, no. 6, pp. 707-715, 2000.

[28] G. Bianchi, A. Banfi, M. Mastrogiacomo et al., "Ex vivo enrichment of mesenchymal cell progenitors by fibroblast growth factor 2," Experimental Cell Research, vol. 287, no. 1, pp. 98-105, 2003.

[29] I. Martin, A. Muraglia, G. Campanile, R. Cancedda, and R. Quarto, "Fibroblast growth factor-2 supports ex vivo expansion and maintenance of osteogenic precursors from human bone marrow," Endocrinology, vol. 138, no. 10, pp. 4456-4462, 1997.

[30] L. A. Solchaga, K. Penick, V. M. Goldberg, A. I. Caplan, and J. F. Welter, "Fibroblast growth factor-2 enhances proliferation and delays loss of chondrogenic potential in human adult bone-marrow-derived mesenchymal stem cells," Tissue Engineering Part A, vol. 16, no. 3, pp. 1009-1019, 2010.

[31] L. A. Solchaga, K. Penick, J. D. Porter, V. M. Goldberg, A. I. Caplan, and J. F. Welter, "FGF-2 enhances the mitotic and chondrogenic potentials of human adult bone marrowderived mesenchymal stem cells," Journal of Cellular Physiology, vol. 203, no. 2, pp. 398-409, 2005.

[32] S. Tsutsumi, A. Shimazu, K. Miyazaki et al., "Retention of multilineage differentiation potential of mesenchymal cells during proliferation in response to FGF," Biochemical and Biophysical Research Communications, vol. 288, no. 2, pp. 413419, 2001.

[33] S. E. Haynesworth, J. Goshima, V. M. Goldberg, and A. I. Caplan, "Characterization of cells with osteogenic potential from human marrow," Bone, vol. 13, no. 1, pp. 81-88, 1992.

[34] D. P. Lennon, S. E. Haynesworth, S. P. Bruder, N. Jaiswal, and A. I. Caplan, "Human and animal mesenchymal progenitor cells from bone marrow: identification of serum for optimal selection and proliferation," In Vitro Cellular and Developmental Biology, vol. 32, no. 10, pp. 602-611, 1996.

[35] D. P. Lennon and A. I. Caplan, "Isolation of human marrowderived mesenchymal stem cells," Experimental Hematology, vol. 34, no. 11, pp. 1604-1605, 2006.

[36] B. Johnstone, T. M. Hering, A. I. Caplan, V. M. Goldberg, and J. U. Yoo, "In vitro chondrogenesis of bone marrow-derived mesenchymal progenitor cells," Experimental Cell Research, vol. 238, no. 1, pp. 265-272, 1998.
[37] A. M. Mackay, S. C. Beck, J. M. Murphy, F. P. Barry, C. O. Chichester, and M. F. Pittenger, "Chondrogenic differentiation of cultured human mesenchymal stem cells from marrow," Tissue Engineering, vol. 4, no. 4, pp. 415-428, 1998.

[38] J. U. Yoo, T. S. Barthel, K. Nishimura et al., "The chondrogenic potential of human bone-marrow-derived mesenchymal progenitor cells," Journal of Bone and Joint Surgery A, vol. 80, no. 12, pp. 1745-1757, 1998.

[39] R. K. Jaiswal, N. Jaiswal, S. P. Bruder, G. Mbalaviele, D. R. Marshak, and M. F. Pittenger, "Adult human mesenchymal stem cell differentiation to the osteogenic or adipogenic lineage is regulated by mitogen-activated protein kinase," Journal of Biological Chemistry, vol. 275, no. 13, pp. 96459652, 2000.

[40] N. Jaiswal, S. E. Haynesworth, A. I. Caplan, and S. P. Bruder, "Osteogenic differentiation of purified, culture-expanded human mesenchymal stem cells in vitro," Journal of Cellular Biochemistry, vol. 64, no. 2, pp. 295-312, 1997.

[41] D. P. Lennon, S. E. Haynesworth, D. M. Arm, M. A. Baber, and A. I. Caplan, "Dilution of human mesenchymal stem cells with dermal fibroblasts and the effects on in vitro and in vivo osteochondrogenesis," Developmental Dynamics, vol. 219, no. 1, pp. 50-62, 2000.

[42] M. F. Pittenger, A. M. Mackay, S. C. Beck et al., "Multilineage potential of adult human mesenchymal stem cells," Science, vol. 284, no. 5411, pp. 143-147, 1999.

[43] C. C. Czerkinsky, L. A. Nilsson, and H. Nygren, "A solidphase enzyme-linked immunospot (ELISPOT) assay for enumeration of specific antibody-secreting cells," Journal of Immunological Methods, vol. 65, no. 1-2, pp. 109-121, 1983.

[44] W. Zhang, R. Caspell, A. Y. Karulin et al., "ELISPOT assays provide reproducible results among different laboratories for T-cell immune monitoring-even in hands of ELISPOTinexperienced investigators," Journal of Immunotoxicology, vol. 6, no. 4, pp. 227-234, 2009.

[45] M. Dominici, K. Le Blanc, I. Mueller et al., "Minimal criteria for defining multipotent mesenchymal stromal cells. The International Society for Cellular Therapy position statement," Cytotherapy, vol. 8, no. 4, pp. 315-317, 2006.

[46] A. Uccelli, L. Moretta, and V. Pistoia, "Mesenchymal stem cells in health and disease," Nature Reviews Immunology, vol. 8, no. 9, pp. 726-736, 2008.

[47] E. Zappia, S. Casazza, E. Pedemonte et al., "Mesenchymal stem cells ameliorate experimental autoimmune encephalomyelitis inducing T-cell anergy," Blood, vol. 106, no. 5, pp. 1755-1761, 2005.

[48] L. Sun, K. Akiyama, H. Zhang et al., "Mesenchymal stem cell transplantation reverses multiorgan dysfunction in systemic lupus erythematosus mice and humans," Stem Cells, vol. 27, no. 6, pp. 1421-1432, 2009.

[49] A. Bacigalupo, M. Valle, M. Podestà et al., "T-cell suppression mediated by mesenchymal stem cells is deficient in patients with severe aplastic anemia," Experimental Hematology, vol. 33, no. 7, pp. 819-827, 2005.

[50] A. I. Caplan, "Adult mesenchymal stem cells for tissue engineering versus regenerative medicine," Journal of Cellular Physiology, vol. 213, no. 2, pp. 341-347, 2007.

[51] P. A. Sotiropoulou, S. A. Perez, M. Salagianni, C. N. Baxevanis, and M. Papamichail, "Characterization of the optimal culture conditions for clinical scale production of human mesenchymal stem cells," Stem Cells, vol. 24, no. 2, pp. 462-471, 2006. 
[52] J. Plumas, L. Chaperot, M. J. Richard, J. P. Molens, J. C. Bensa, and M. C. Favrot, "Mesenchymal stem cells induce apoptosis of activated T cells," Leukemia, vol. 19, no. 9, pp. 1597-1604, 2005.

[53] J. Larghero, D. Farge, A. Braccini et al., "Phenotypical and functional characteristics of in vitro expanded bone marrow mesenchymal stem cells from patients with systemic sclerosis," Annals of the Rheumatic Diseases, vol. 67, no. 4, pp. 443-449, 2008.

[54] P. A. Conget and J. J. Minguell, "Phenotypical and functional properties of human bone marrow mesenchymal progenitor cells," Journal of Cellular Physiology, vol. 181, no. 1, pp. 67-73, 1999.

[55] M. D. Nicola, C. Carlo-Stella, M. Magni et al., "Human bone marrow stromal cells suppress T-lymphocyte proliferation induced by cellular or nonspecific mitogenic stimuli," Blood, vol. 99, no. 10, pp. 3838-3843, 2002.

[56] K. Le Blanc, L. Tammik, B. Sundberg, S. E. Haynesworth, and O. Ringdén, "Mesenchymal stem cells inhibit and stimulate mixed lymphocyte cultures and mitogenic responses independently of the major histocompatibility complex," Scandinavian Journal of Immunology, vol. 57, no. 1, pp. 11-20, 2003.

[57] H. Lazarus, P. Curtin, S. Devine, P. McCarthy, K. Holland, and A. Moseley, "Role of mesenchymal stem cells in allogeneic transplantation: early phase I clinical results," Blood, vol. 96, p. 392a, 2000.

[58] H. Samuelsson, O. Ringden, H. Lonnies, and K. L. Blanc, "Optimizing in vitro conditions for immunomodulation and expansion of mesenchymal stromal cells," Cytotherapy, vol. 11, no. 2, pp. 129-136, 2009.

[59] S. E. Haynesworth, J. Goshima, V. M. Goldberg, and A. I. Caplan, "Characterization of cells with osteogenic potential from human marrow," Bone, vol. 13, no. 1, pp. 81-88, 1992.

[60] M. Mastrogiacomo, R. Cancedda, and R. Quarto, "Effect of different growth factors on the chondrogenic potential of human bone marrow stromal cells," Osteoarthritis and Cartilage, vol. 9, pp. S36-S40, 2001.

[61] R. Meisel, A. Zibert, M. Laryea, U. Göbel, W. Däubener, and D. Dilloo, "Human bone marrow stromal cells inhibit allogeneic T-cell responses by indoleamine 2,3-dioxygenasemediated tryptophan degradation," Blood, vol. 103, no. 12, pp. 4619-4621, 2004.

[62] S. Aggarwal and M. F. Pittenger, "Human mesenchymal stem cells modulate allogeneic immune cell responses," Blood, vol. 105, no. 4, pp. 1815-1822, 2005.

[63] M. E. Groh, B. Maitra, E. Szekely, and O. N. Koç, "Human mesenchymal stem cells require monocyte-mediated activation to suppress alloreactive T cells," Experimental Hematology, vol. 33, no. 8, pp. 928-934, 2005.

[64] M. Krampera, L. Cosmi, R. Angeli et al., "Role for interferon$\gamma$ in the immunomodulatory activity of human bone marrow mesenchymal stem cells," Stem Cells, vol. 24, no. 2, pp. 386398, 2006.

[65] J. J. Auletta, K. R. Cooke, L. A. Solchaga, R. J. Deans, and W. van't Hof, "Regenerative stromal cell therapy in allogeneic hematopoietic stem cell transplantation: current impact and future directions," Biology of Blood and Marrow Transplantation, vol. 16, no. 7, pp. 891-906, 2010. 

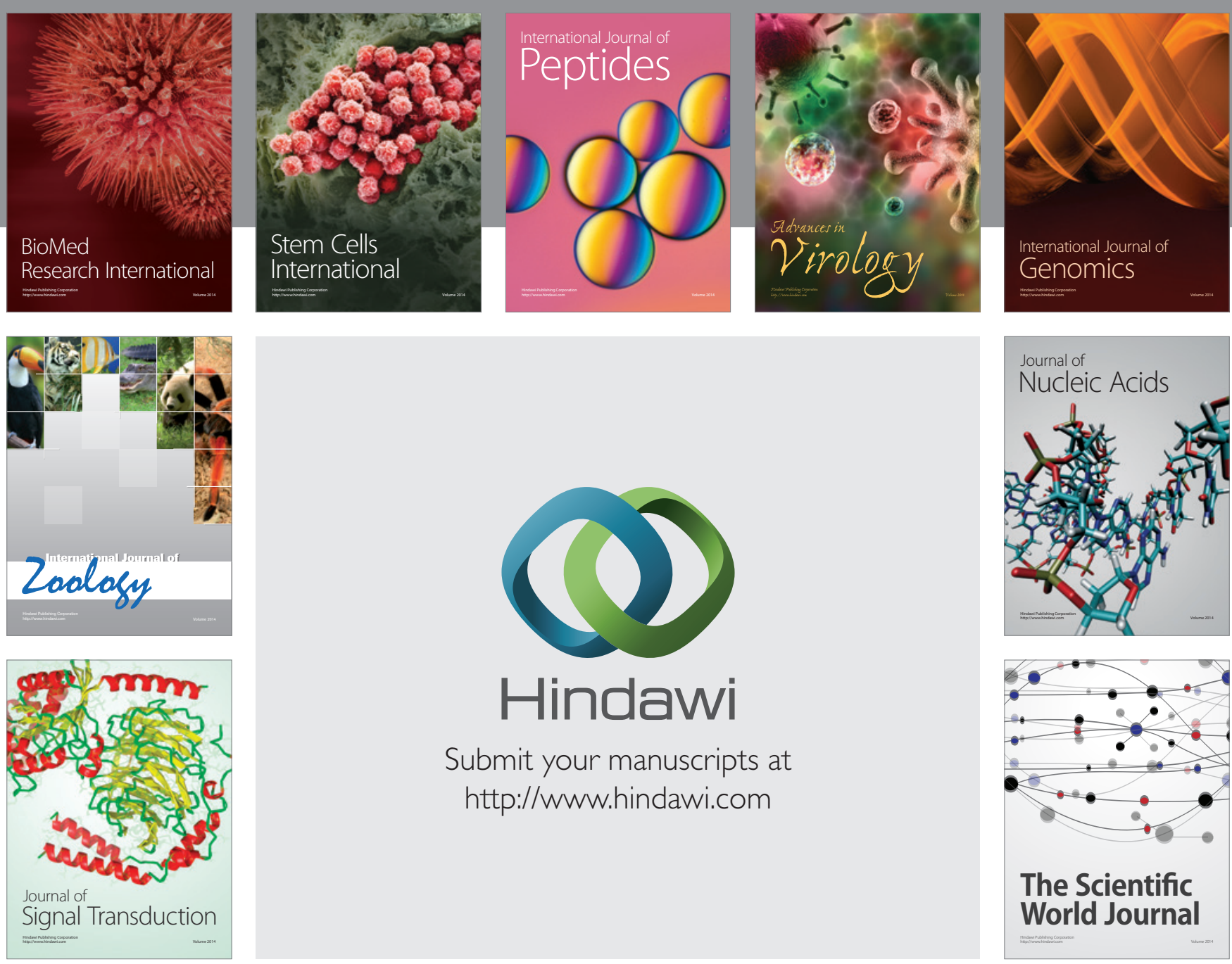

Submit your manuscripts at

http://www.hindawi.com
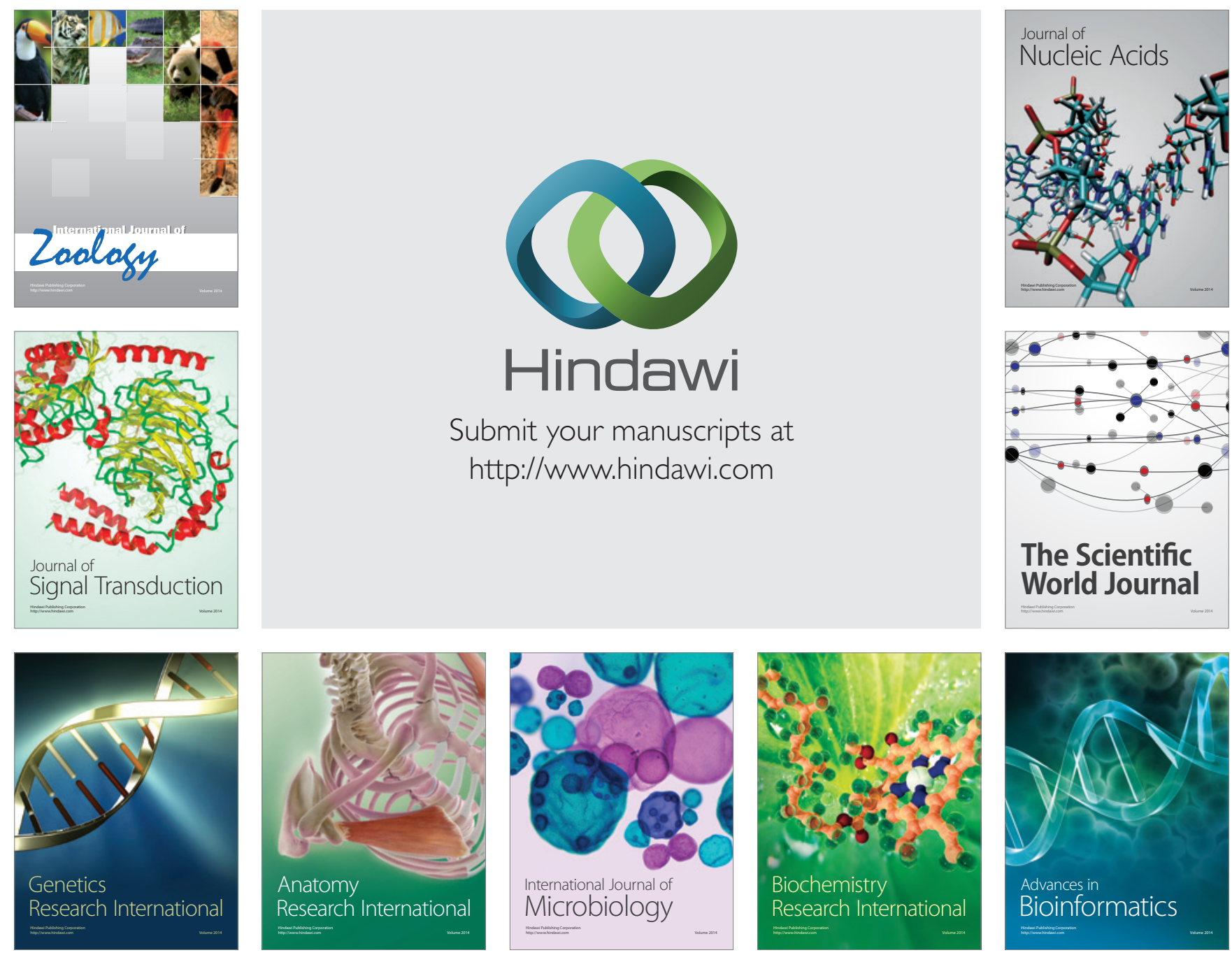

The Scientific World Journal
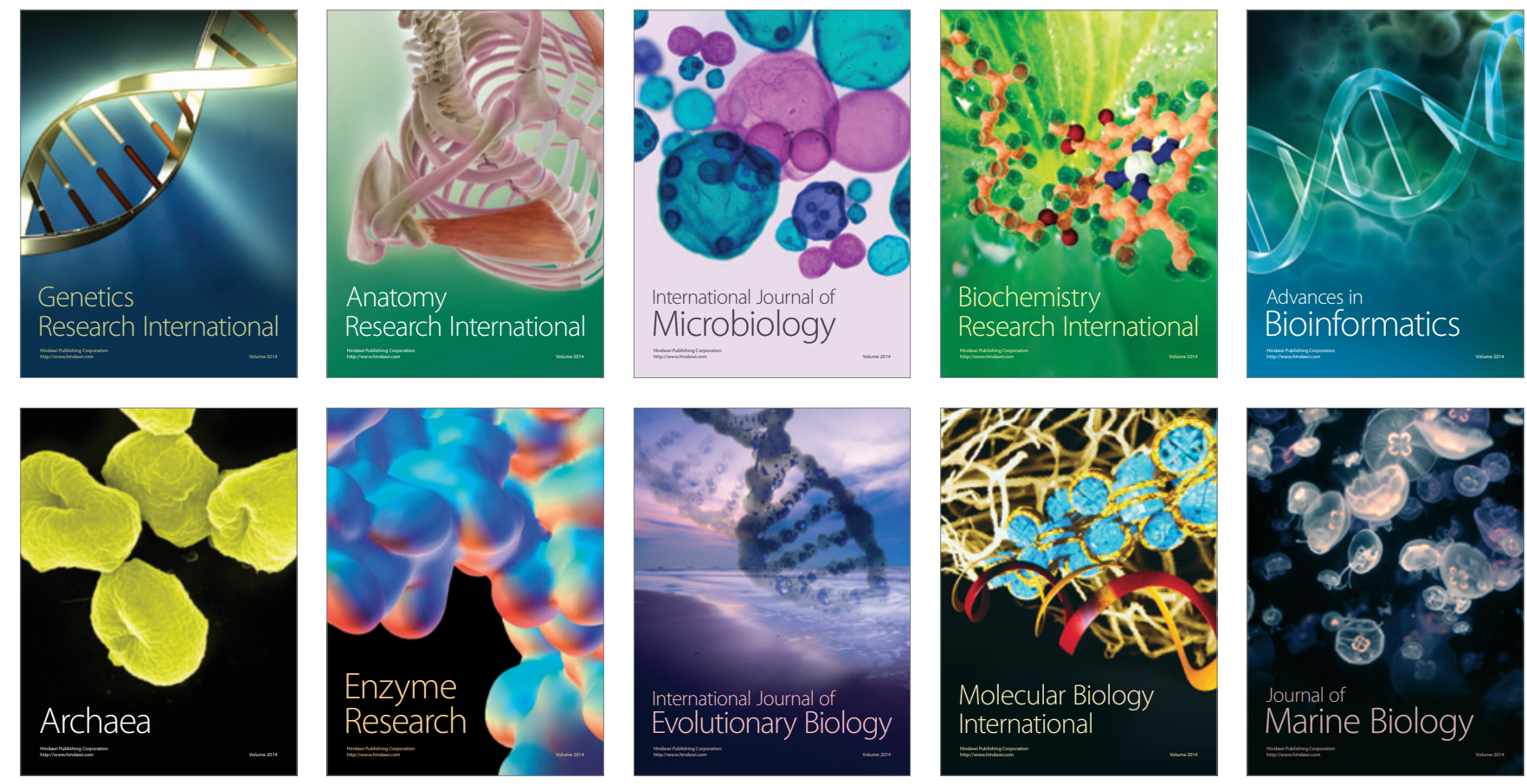